


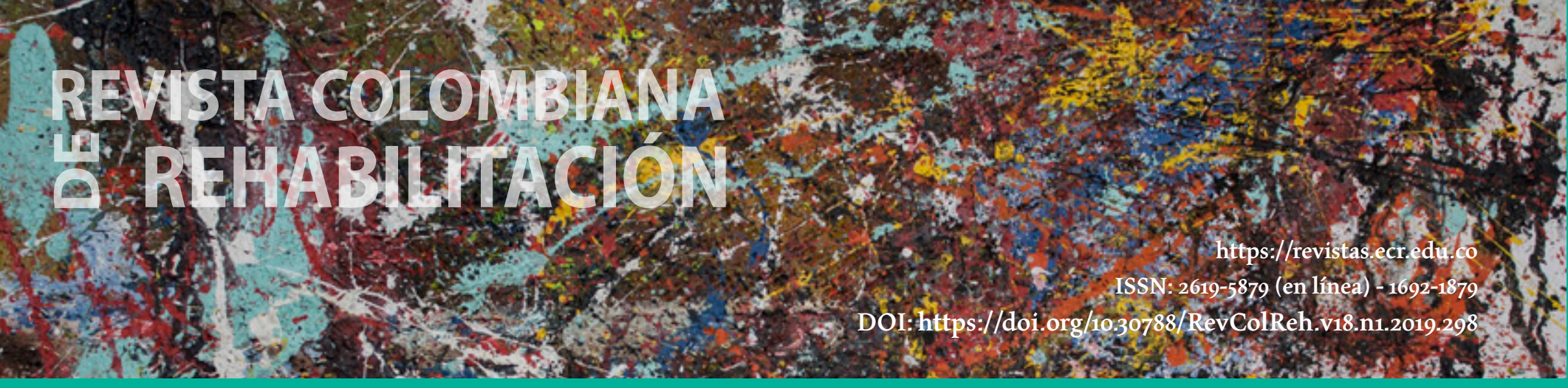

Fecha de recepción: 10 de mayo de 2018 Fecha de aprobación: 16 de noviembre de 2018

\title{
Nivel de actividad física en mujeres con cáncer de mama no metastásico de dos instituciones de salud de alta complejidad
}

\author{
Paola Andrea Córdoba Barona ${ }^{\mathrm{a}}$ \\ https://orcid.org/oooo-0002-2636-6612
}

Esther Cecilia Wilches-Lunac
https://orcid.org/oooo-0003-3255-7607

\author{
Viviana Carolina López López \\ https://orcid.org/oooo-0oo2-4560-1120
}

Angelly Bustamente ${ }^{c}$

https://orcid.org/oooo-0003-3898-0879

\author{
Gina Paola Velasco Villaní \\ https://orcid.org/oooo-0002-5695-5354 \\ a. Fundación Clínica Valle de Lili, Cali, Colombia \\ b. Christus Sinergia Clínica Farallones, Cali, Colombia \\ c. Universidad del Valle, Cali, Colombia
}

\section{Resumen}

La incidencia del cáncer de mama va en aumento progresivo durante las últimas décadas, y para el año 2020 podría aumentar en un 50\%. La actividad física en esta población actúa como un factor protector para la salud. Objetivo: describir los niveles de actividad física en mujeres con cáncer de mama no metastásico reportadas en bases de datos de dos Instituciones de salud de la ciudad de Cali (Colombia) durante un año. Método: Estudio observacional descriptivo de corte transversal con 30 mujeres adultas, la recolección de información se realizó mediante el Cuestionario Internacional de Actividad Física versión corta (IPAQ-SF) y un cuestionario de datos generales, se relacionó el nivel de actividad física con el estadio de la enfermedad, la ocupación, el nivel socioeconómico, el índice de masa corporal (IMC) y la edad. Resultados: el 30,o\% presentó nivel alto de actividad física, el 33,3\% nivel moderado y el 36, 7\% nivel bajo; además, el 83\% de las mujeres presentaron sobrepeso. Se evidenció una asociación estadísticamente significativa entre el estadio de cáncer y actividad física $(\mathrm{p}=\mathrm{0}, \mathrm{014})$. Discusión: Los resultados son coherentes con la literatura en lo relacionado con la evaluación de la actividad física a través del IPAQ en mujeres con cáncer de mama, los niveles de actividad física en estas sobrevivientes son generalmente bajos. El incremento en los niveles de actividad física en personas con Ca de mama es una tanto individual como social. Se requiere una perspectiva poblacional, multisectorial, multidisciplinaria, y culturalmente idónea.

Palabras clave: Fisioterapia; ejercicio; deporte; perfil laboral

\section{Level of physical activity in women with non-metastasic breast cancer of two institutions is health of high complexity}

\begin{abstract}
The incidence of breast cancer has progressively increased during the last decades and by the year 2020 it could increase by $50 \%$. Physical activity in the population suffering it acts as a protective factor for health. Objective: The purpose of the study was to describe the levels of physical activity among women with non-metastatic breast cancer, as reported in the databases of two health institutions of the city of Cali through a year. Method: An observational descriptive study was conducted on a sample of 30 women. They were asked to complete The International Physical Activity Questionnaire - Short Form (IPAQ-SF) and a questionnaire with general inquiries. The data was collected and analyzed relating the physical activity level, the cancer stage, the patient's occupation, their socioeconomic level, their body mass index (BMI) and their age. Results: The results revealed that $30 \%$ of the population kept their physical activity at a high level, $33.3 \%$ at a moderate level and $36.7 \%$ at a low level. The results also disclosed that $83 \%$ of women in the sample were overweight and that there was a statistically significant correlation between cancer stages and physical activity $(\mathrm{p}=0.014)$. Discussion: The results are consistent with the literature relating the evaluation of physical activity through the IPAQ in women with breast cancer: their level of physical activity is generally low. The increase in physical activity levels in people with breast cancer is both individual and social. A demographic, multisector, multidisciplinary, and culturally appropriate perspective is required.
\end{abstract}

Keywords: Breast, breast neoplasms; exercise; physical activity. 


\section{Introducción}

1 l cáncer de mama es un problema de salud pública a nivel mundial, debido a que es la primera causa de muerte en la población femenina.(González-Robledo, González-Robledo, Nigenda, \& López-Carrillo, 2010) y el tumor más común en el mundo (Akram, Iqbal, Daniyal, \& Khan, 2017). En Colombia se encuentra entre las primeras diez causas de muerte contribuyendo en un $15 \%$ en la mortalidad de mujeres y en Cali ocupa el primer lugar ( «Cali modelo de atención de cáncer de mama», s. f.); según el Registro Poblacional de cáncer de Cali en el periodo 20o82012 la tasa de incidencia media anual por cada 100.000 mujeres correspondió a 2893 casos con una frecuencia relativa del $24.33 \%$ y en el periodo 2011- 2015 presento una tasa de mortalidad media anual de 1054 casos con una frecuencia relativa de 15.67\%. («Registro Poblacional de Cáncer de Cali», s. f.).

La incidencia de ésta enfermedad ha aumentado progresivamente en las últimas décadas debido a diversos factores como la inactividad física, exposición a hormonas endógenas y exógenas, entre otros (Hacker, 2009). La Organización Mundial de la Salud (OMS) plantea en su informe que la incidencia de ésta podría aumentar en un 50\% hasta el año 2020, en el que habría 15 millones de nuevos casos.(«OMS | La incidencia mundial del cáncer podría aumentar en un 50\% y llegar a 15 millones de nuevos casos en el año 2020», s. f.)

La condición física y funcional son factores que se ven afectados en personas que padecen cáncer debido a la patología especifica o al tratamiento al cual sean sometidos, además factores como la edad y la condición de salud previa también influyen en la condición actual de la persona (Hacker, 2009).

La realización de actividad física en esta población actúa como un factor protector para la salud, debido a que se ha evidenciado beneficios no solo del bienestar físico sino también del psicológico. No obstante, se ha identificado que los niveles de actividad física en mujeres con diagnóstico de cáncer de mama suelen ser bajos como efecto secundario del empleo de los diversos tratamientos.(Mutrie et al., 2007; Ramírez, Acevedo, Herrera, Ibáñez, \& Sánchez, 2017; VardarYagli et al., 2015). De igual manera algunos autores analizan la experiencia de las mujeres con cáncer de mama en torno a la enfermedad, los tratamientos y el malestar psicológico (Belber-Gómez, Valencia-Agudo, \& Viuda-Suárez, 2018), lo que también podría comprometer los niveles de actividad física.

Los niveles altos de actividad física en mujeres con cáncer de mama contribuyen a una mejor respuesta al tratamiento y minimizan los efectos secundarios (Alfano et al., 2007). Por lo que la inactividad física es considerada como un factor de riesgo para el cáncer de mama y la reincidencia del mismo. Importancia que resalta también en el Plan Decenal para el Control de Cáncer en Colombia $(2012$ - 2021) del año 2013 el cual contempla en sus lineamientos estratégicos, la promoción de la actividad física como control del riesgo, enmarcada en la prevención primaria.

Existe en la literatura evidencia relacionada con el uso del Cuestionario Internacional de Actividad Física (IPAQ), que ha sido ampliamente utilizado para evaluar los niveles de actividad física en mujeres con cáncer (Ca) de mama y que cuenta con versión validada en población colombiana (Cuevas et al., 2014; Kang et al., 2017; Mohammadi, Sulaiman, Koon, Amani, \& Hosseini, 2013; Ortiz et al., 2018; Sabino Neto, Moreira, Resende, \& Ferreira, 2012; Vardar-Yagli et al., 2015)

Debido a que a nivel local no se conocen reportes que describan la relación entre los niveles de actividad física y el Ca de mama en mujeres, se planteó como objetivo de ésta investigación describir los niveles de actividad física en mujeres con cáncer de mama no metastásico reportadas en las bases de datos de dos instituciones de salud de la ciudad de Cali en el periodo abril-junio de 2014 .

\section{Método}

Se realizó un estudio observacional descriptivo de corte transversal, en la ciudad de Cali (Colombia) en un período de tres meses, con la utilización de bases de datos de dos Instituciones de salud de la ciudad.

\section{Participantes}

Participaron en el estudio todas las mujeres que cumplieron los criterios de inclusión y que aceptaron participar. Se 
calculó el tamaño de muestra utilizando el software Epiinfo, versión 3.5. Inicialmente se calculó el tamaño de muestra de 80 mujeres con base en $5 \%$ de precisión, 50\% de prevalencia de fatiga en esta población (se utilizó el máximo valor de prevalencia debido a la alta variabilidad de este dato en los estudios revisados y corrección por no respuesta de $15 \%$ ). Posteriormente debido a las dificultades del reclutamiento de las mujeres, se recalculo el tamaño de muestra, teniendo en cuenta la prevalencia reportada en la literatura y se aumentó el error muestral, los parámetros finales fueron $n=40$ y el cálculo se realizó mediante la fórmula que se presenta en la figura 1.

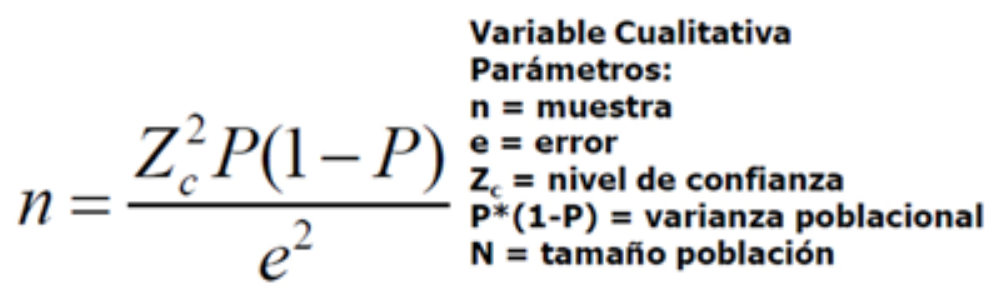

Figura 1. Fórmula para el cálculo de la muestra

Los criterios de inclusión contemplados en el estudio fueron mujeres con edad entre los 22 y 65 años, con diagnóstico médico de Ca de mama que hubieren terminado tratamiento y que accedieran a participar en el estudio después de firmar el consentimiento informado, los criterios de exclusión fueron mujeres con Ca metastásico y/o que recibieran cuidado paliativo, con diagnóstico clínico de enfermedad mental y que recibieran tratamiento para éste.

El estudio tomó como base el tamaño muestral mencionado y se reclutó una muestra por conveniencia de 30 mujeres hasta la fecha de finalización de la recolección de datos. Este grupo de mujeres correspondieron al 75\% de la muestra original. En la Figura 2. Se muestran las mujeres incluidas en la investigación.

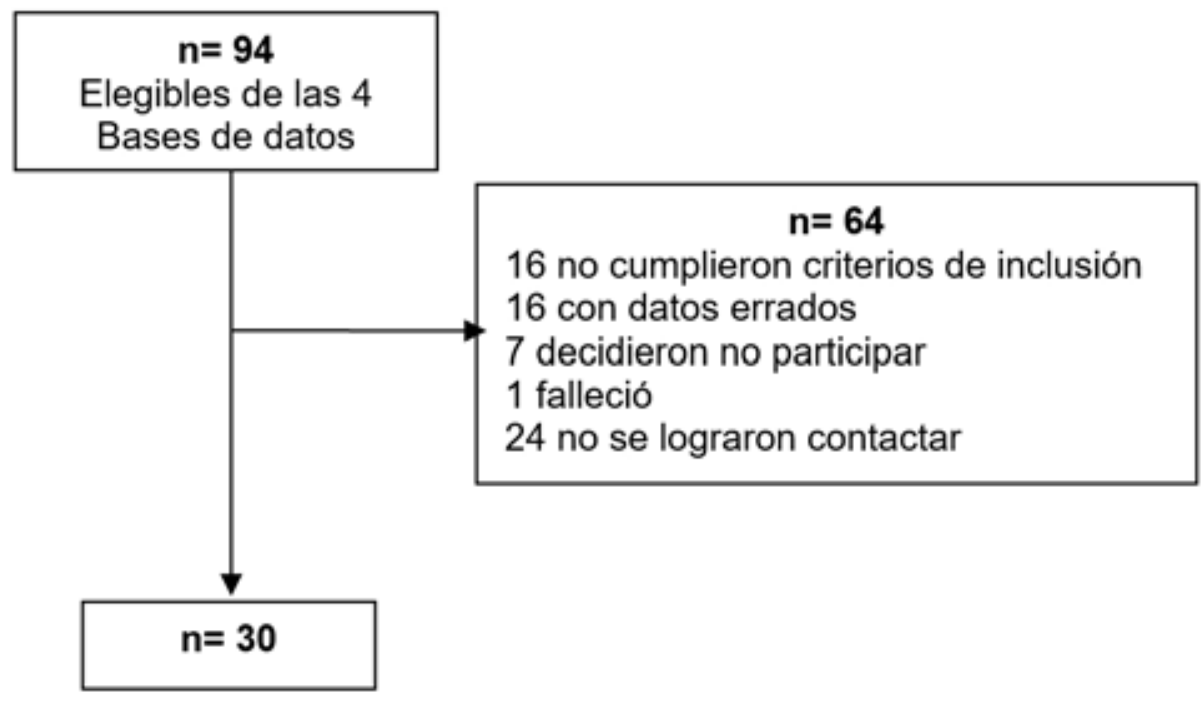

Figura 2. Selección y número de mujeres participantes en el estudio

\section{Instrumentos}

Para evaluar el nivel de actividad física se utilizó el Cuestionario Internacional de Actividad Física versión corta (IPAQ-SF por sus siglas en inglés), el cual es un instrumento diseñado principalmente para la vigilancia de la población 
en cuanto a la actividad física entre los adultos. Se ha desarrollado y probado para su uso en adultos (rango de edad de 15 a 69 años). (Fernández, Sánchez, \& Hermoso, 2005)

La adaptación cultural de la versión para Colombia se realizó mediante una revisión inicial de la versión del formato telefónico corto en español para Estados Unidos, a partir del cual se llevó a cabo la adaptación cultural del instrumento. En este proceso se tuvo en cuenta el contexto social de la población urbana de estratos socioeconómicos bajos y medios de Colombia, que representa la mayor proporción de habitantes del país.(«Downloadable questionnaires - International Physical Activity Questionnaire», s. f.)

Este instrumento evalúa la actividad física realizada e incluye las siguientes categorías: a) actividad física en el tiempo libre, b) actividades en la casa, domésticas y de jardín (patio), c) actividad física relacionada con el trabajo y d) actividad física relacionada con el transporte (Fernández et al., 2005).La versión corta de IPAQ, pregunta sobre 3 tipos de actividad como resultado de las cuatro áreas anteriores, los tipos específicos de actividad son: a) andar, b) actividades de intensidad moderada y c) actividades de intensidad vigorosa. El instrumento está estructurado para proporcionar resultados independientes para estos tres tipos de actividad (Fernández et al., 2005).

La obtención del resultado total para la versión corta requiere la suma de la duración (en minutos) y de la frecuencia (días) de estos 3 tipos de actividad. Los resultados específicos para cada una de las áreas no pueden ser estimados en la versión corta (Fernández et al., 2005). El nivel de actividad física se clasificó de acuerdo con los MET (Equivalente Metabólico como baja (menor de $600 \mathrm{MET}$ ), moderada (600 y $1500 \mathrm{MET}$ ) y alta (Mayor de 1500 MET).(Fernández et al., 2005)

Se utilizó un formato de recolección de datos, que incluyó aspectos relacionados con edad, ocupación, estadio de la enfermedad y estrato socioeconómico. El peso $(\mathrm{kg})$ y la estatura $(\mathrm{cm})$ se midieron con técnicas estandarizadas, con los participantes en ropa ligera y sin zapatos. Las mediciones se realizaron con una báscula calibrada (o,1 kg de precisión) (Health o Meter Professional ${ }^{\circledR}$ ) (RANGO). La báscula, con capacidad de $350 \mathrm{lb} / 160 \mathrm{~kg}$ y una barra de medición o Estadiómetro (o,5 cm de precisión) con rangos de 60 a $213 \mathrm{~cm}$. Con estas variables se calculó el IMC en $\mathrm{Kg} / \mathrm{m} 2$.

Se realizó una prueba piloto con la participación de 5 mujeres sin Ca de mama, que se encontraban en el rango de edad contemplado en los criterios de inclusión, Los resultados, permitieron identificar la necesidad de cambiar la forma de aplicación del cuestionario IPAQ-SF, aplicándolo de manera dirigida y no auto-administrada, debido a la confusión presentada por las participantes para el diligenciamiento del cuestionario. El cuestionario se aplicó de manera dirigida y con una duración de entre 20 y 30 minutos.

\section{Procedimiento}

Como parte del macroproyecto: "Relación entre fatiga y capacidad aeróbica en mujeres con cáncer de mama no metastásico en una institución de salud de alta complejidad del valle del cauca en el 2013-2014”, se estableció contacto con el personal de salud de dos instituciones, especialmente con las enfermeras jefes de las unidades de quimioterapia y radioterapia obteniendo las bases de datos de las mujeres con diagnóstico de cáncer de mama.

Con la información obtenida, se seleccionaron las mujeres que cumplieron con los criterios de inclusión, posteriormente se realizó contacto telefónico, en el cual se corroboró la información y se explicaron los objetivos, beneficios, riesgos y duración del estudio, con las mujeres que aceptaron participar, se acordaron citas de evaluación teniendo en cuenta el cronograma establecido y la disponibilidad de las mismas.

Una vez aceptada la participación en el estudio con la firma del consentimiento informado, el día de la evaluación, se diligenció el formato de recolección de datos, luego se realizó la medición de peso y talla y por último se procedió a diligenciar de manera dirigida el Cuestionario IPAQ-SF. A cada formato de recolección de datos se le asignó un código (número de registro del cuestionario de recolección de datos del o1 al 30).

Al completar la evaluación se enviaba a cada participante vía correo electrónico, de manera individualizada los resultados con una clara explicación.

La información fue digitada en una base datos en Excel, la cual se procesó usando el software Epiinfo, versión 3.5. Se realizó un análisis bivariado con el fin de identificar posibles asociaciones entre las variables relacionadas con las carac- 
terísticas sociodemográficas, el estadío del cáncer y el índice de masa corporal y el nivel de actividad física, para ello la variable nivel de actividad física se recategorizó, éste análisis fue realizado a través de la prueba Chiz.

Este estudio fue aprobado por el Comité de Ética de la Universidad del Valle-Cali Colombia (Acta de aprobación 081-014) y de las instituciones de salud donde se revisaron las bases de datos; fue clasificado como una investigación sin riesgo de acuerdo a la resolución oo8430 de 1993 del Ministerio de Protección Social. Todos los sujetos incluidos en el estudio firmaron el Consentimiento Informado y se hizo entrega de una copia de este a cada participante.

\section{Resultados}

Del total de mujeres evaluadas ( $\mathrm{n}=30$ ) se observó que el 73 \% (22) pertenecían al régimen de salud contributivo, en cuanto al estado civil el 33 \% (10 mujeres) vivían en unión libre; al analizar la ocupación el 53\% (16) son amas de casa, mientras que 37 \% (11) trabajan, con respecto al estrato socioeconómico el 36 \% (11 mujeres) pertenecen a estrato 2. El promedio de edad de las mujeres incluidas en el estudio fue de 56 años con una desviación estándar de 6,24 años como se indica en la tabla 1.

El estadio de cáncer más frecuente fueron los estadios 1 y 2; el peso promedio fue de $73 \mathrm{~kg}$, el peso mínimo fue $43 \mathrm{~kg}$, el $50 \%$ de las mujeres incluidas en el estudio presentaban un peso por debajo de $74 \mathrm{~kg}$, en cuanto a la talla se evidencia un promedio de $158,3 \mathrm{~cm}$, con una desviación de $5,39 \mathrm{~cm}$, el $50 \%$ presentaron una talla por debajo de $157,5 \mathrm{~cm}$.

$\mathrm{Al}$ analizar el IMC, se encontró que el 83,3 \% de las mujeres incluidas en el estudio presentaban sobrepeso, y el 13,3\% peso normal como se indica en la Tabla 1.

\section{Tabla 1.}

Estadística descriptiva de las variables sociodemográficas y de salud de las mujeres participantes en el estudio ( $n=30$ ).

\begin{tabular}{|c|c|}
\hline Categorías & Mujeres n (\%) \\
\hline \multicolumn{2}{|c|}{ Régimen de Salud } \\
\hline Contributivo & $22(73)$ \\
\hline Subsidiado & $8(27)$ \\
\hline \multicolumn{2}{|l|}{ Estado Civil } \\
\hline Unión Libre & $10(33)$ \\
\hline Casada & $8(27)$ \\
\hline Separada & $3(10)$ \\
\hline Soltera & $3(10)$ \\
\hline Viuda & $3(10)$ \\
\hline Divorciada & $2(7)$ \\
\hline No respondió & $1(3)$ \\
\hline \multicolumn{2}{|l|}{ Ocupación } \\
\hline Ama de Casa & $16(53)$ \\
\hline Trabaja & $11(37)$ \\
\hline Otro & $2(7)$ \\
\hline Desempleada & $1(3)$ \\
\hline \multicolumn{2}{|l|}{ Estrato } \\
\hline 1 & $4(13)$ \\
\hline 2 & $11(36)$ \\
\hline 3 & $10(33)$ \\
\hline 4 & $3(10)$ \\
\hline 5 & $2(7)$ \\
\hline Edad & $\begin{array}{l}\chi \sim=56 \\
D E=6,24\end{array}$ \\
\hline
\end{tabular}

\section{Estadio del cáncer}




\begin{tabular}{ll}
\hline Categorías & Mujeres $\mathrm{n}(\%)$ \\
\hline Estadio 2 & $14(47)$ \\
Estadio 3 & $6(20)$ \\
\hline Índice de masa corporal & \\
Bajo Peso & $1(3,3)$ \\
Peso Normal & $4(13,3)$ \\
Sobrepeso & $25(83,3)$ \\
\hline
\end{tabular}

La relación entre niveles de actividad física y el estadio de cáncer puede observarse en la figura 3. De acuerdo con los resultados del IPAQ-SF, 30\% de mujeres registraron un nivel alto de actividad física, 33,3\% nivel moderado y $36.7 \%$ nivel bajo. El análisis mediante prueba Chi2 mostró una asociación estadísticamente significativa $(p=0,014)$

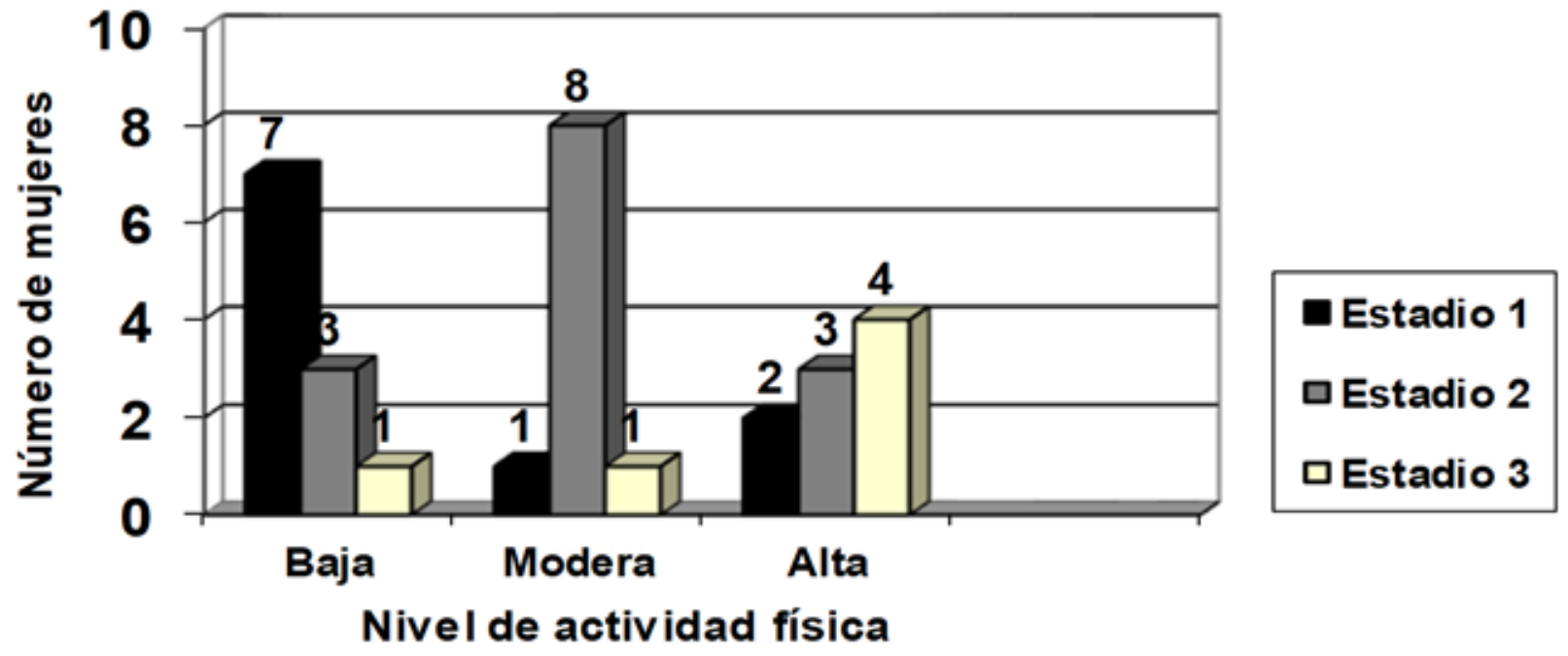

Figura 3. Relación entre el nivel de actividad física y el estadio del cáncer

Al analizar la ocupación, el estrato socioeconómico, el IMC y la edad con el nivel de actividad física, no se observaron asociaciones estadísticamente significativas entre estas variables $(\mathrm{p}=0,419, \mathrm{p}=0,214$ y $\mathrm{p}=0,603$ y p o,804) respectivamente.

\section{Discusión}

Este estudio es pionero en nuestro medio en la evaluación de los niveles de actividad física en mujeres con Ca de mama. El 63.3\% de las mujeres presentaron niveles de actividad física entre alto y moderado, resultados que difieren de otro estudio realizado donde analizaron la relación entre la actividad física, la discapacidad, y el perfil de la condición física de mujeres latinas sobrevivientes de cáncer de mama el cual encontró niveles bajos de actividad y condición física. (Ortiz et al., 2018)

Los autores de la presente investigación asumen, que los niveles de actividad física altos y moderados podrían estar relacionados con que el $53 \%$ de las participantes realizaban actividades domésticas y el $37 \%$ trabajo fuera de casa, lo que de alguna manera implicaba para ellas realizar algún tipo de actividad física.

La Organización Mundial de la Salud (OMS) plantea en sus recomendaciones sobre actividad física en el rango de edad de 18 a 64 años, "una dedicación mínima de 150 minutos semanales a la práctica de actividad física aeróbica de intensidad moderada, o bien 75 minutos de actividad física aeróbica vigorosa cada semana o bien una combinación equivalente de actividades moderadas y vigorosas". En este estudio solo el 63,3\% (19/30) cumplía con la recomendación cuando se combinaba el tiempo de actividades físicas moderadas y vigorosas. («OMS | Recomendaciones mundiales sobre la actividad física para la salud», s. f.)

La falta de ejercicio físico parece asociarse con ganancia de peso durante la supervivencia al Ca de mama, (Holmes, 
Chen, Feskanich, Kroenke, \& Colditz, 2005) ya que tanto el sobrepeso en el momento del diagnóstico del cáncer como la ganancia de peso luego de éste están asociados con menor supervivencia. En este estudio 83\% (24/30) de las mujeres evaluadas fueron clasificadas con sobrepeso de acuerdo a la clasificación de la OMS, datos similares a los reportados en otro estudio (Amaral et al., 2010) quienes realizaron un estudio en donde analizaron la grasa corporal y mala alimentación en 71 mujeres con cáncer de mama, encontrando una amplia prevalencia de sobrepeso (34\%) y obesidad (18\%) y una cantidad excesiva de grasa corporal y abdominal tras el diagnóstico de cáncer de mama.

Aumentar el nivel de actividad física es una necesidad social, no solo individual. Por lo tanto, exige una perspectiva poblacional, multisectorial, multidisciplinaria, y culturalmente idónea. Diferentes publicaciones realizadas refieren la implementación de la valoración de la actividad física a través del IPAQ en mujeres con cáncer de mama; y sus resultados revelan que los niveles de actividad física en las sobrevivientes de cáncer de mama son generalmente bajos. (Fernández et al., 2005; Furmaniak, Menig, \& Markes, 2016; Irwin et al., 2004; Kang et al., 2017)

Un estudio evaluó los factores asociados con la actividad física o la inactividad en 3088 mujeres sobrevivientes de Ca de mama, mostrando que las variables demográficas y psicosociales se relacionaron con los niveles de actividad física ( $p$ $<0,001$ ). (Irwin et al., 2007) Lo anterior, difiere con los resultados de este estudio, en donde no se encontraron diferencias significativas, cuando se relacionaron los niveles de actividad fisca con la edad ( $p=0,804)$, ocupación ( $p=0,419)$, y con el estrato socio económico $(\mathrm{p}=0,214)$.

Las participantes tuvieron un promedio de edad de 56 años (SD 6,24 años); debido a la gran diferencia en la edad de las mujeres incluidas en los artículos revisados no fue posible realizar comparaciones al respecto.

Existen otros aspectos de gran relevancia respecto el Ca de mama como la variedad histológica (tipo) del tumor y su etapa o estadio; el estadio hace referencia a la extensión o gravedad del cáncer que aqueja a un individuo con base en la extensión del tumor original (primario) y la extensión de la diseminación en el cuerpo. (Salas Zapata \& Grisales Romero, 2010) En este estudio el 47\% (14/30) de las sobrevivientes de cáncer de mama, se encontraban en estadio II y los hallazgos mostraron una asociación estadísticamente significativa entre el estadio del cáncer y actividad física $(\mathrm{p}=0,014)$; con una relación directamente proporcional; a diferencia de lo reportado en un estudio realizado, donde la relación fue inversamente proporcional (Furmaniak et al., 2016) lo que podría estar influenciado por los tratamientos como la quimioterapia y radioterapia los cuales tienen repercusión a nivel cardiovascular con efectos a nivel cardiaco y en la capacidad aeróbica, esto se traduce en abandono de la actividad física con consecuencias como la fatiga y la descalcificación ósea. (Holmes et al., 2005)

Los autores reconocen como limitaciones del estudio, las dificultades en el reclutamiento de las voluntarias, como también las relacionadas con la edad, el tiempo de finalización del tratamiento y la ausencia de información relacionada con la quimioterapia y radioterapia, lo cual no permitió extrapolar los resultados a todas las mujeres sobrevivientes de Ca de mama de la ciudad de Cali.

\section{Implicaciones Clínicas}

Este estudio brinda un marco referencial en el medio, relacionado con los niveles de actividad física en mujeres con Ca de mama, información desconocida a la fecha por los servicios de salud, los cuales deben tener en cuenta la importancia de estandarizar la evaluación de los niveles de actividad física, en las sobrevivientes de Ca de mama, de tal manera que se puedan identificar las necesidades individuales con el fin de ofrecer una atención integral que permita el mejoramiento de la salud en esta población.

Se recomienda realizar futuros estudios con mayor tamaño muestral y otro tipo de diseño metodológico, que además de evaluar el nivel de actividad física, también analicen otras variables clínicas e intervenciones que contribuyan a establecer mejor la relación de la actividad física y el estadio del cáncer de mama.

\section{Agradecimientos}

A las mujeres e Instituciones de salud participes del estudio. 


\section{Conflicto de Intereses}

Las autoras declaran no tener ningún conflicto de interés en la elaboración y publicación del presente trabajo.

\section{Colaboraciones}

Todas las autoras han contribuido intelectualmente en la elaboración del documento. 
Akram, M., Iqbal, M., Daniyal, M., \& Khan, A. U. (2017). Awareness and current knowledge of breast cancer. Biological Research, 50(1), 33. https://doi.org/10.1186/s40659-017-0140-9

Alfano, C. M., Smith, A. W., Irwin, M. L., Bowen, D. J., Sorensen, B., Reeve, B. B., ... McTiernan, A. (2007). Physical activity, long-term symptoms, and physical health-related quality of life among breast cancer survivors: A prospective analysis. Journal of Cancer Survivorship, 1(2), 116. https://doi.org/10.1007/s11764-007-0014-1

Amaral, P., Miguel, R., Mehdad, A., Cruz, C., Monteiro Grillo, I., Camilo, M., \& Ravasco, P. (2010). Body fat and poor diet in breast cancer women. Nutricion Hospitalaria, 25(3), 456-461. Recuperado de http://scielo.isciii.es/scielo. php?script=sci_arttext\&pid=So212-16112010000300018\&lng=en\&nrm=iso\&tlng=en

Belber-Gómez, M., Valencia-Agudo, F., \& Viuda-Suárez, M. E. de la. (2018). Análisis de la experiencia subjetiva en mujeres con cáncer de mama. Duazary: Revista Internacional de Ciencias de La Salud, 15(1), 71-85. https://doi.org/10.21676/2389783X.2021

Cali modelo de atención de cáncer de mama. (s. f.). Recuperado 7 de mayo de 2018, de http://www.cali.gov.co/publicaciones/31659/cali_modelo_de_atencin_de_cncer_de_mama/

Cuevas, B. T., Hughes, D. C., Parma, D. L., Treviño-Whitaker, R. A., Ghosh, S., Li, R., \& Ramirez, A. G. (2014). Motivation, exercise, and stress in breast cancer survivors. Supportive Care in Cancer, 22(4), 911-917. https://doi.org/10.1007/s00520-013-2038-6

Downloadable questionnaires - International Physical Activity Questionnaire. (s. f.). Recuperado 8 de noviembre de 2018, de https://sites.google.com/site/theipaq/questionnaire_links

Fernández, M. D., Sánchez, P. T., \& Hermoso, V. M. S. (2005). Traducción de la Guía para el procesamiento de datos y análisis del cuestionario internacional de actividad física (IPAQ). Versiones corta y larga. Universidad de Granada. Junta de Andalucía.

Furmaniak, A. C., Menig, M., \& Markes, M. H. (2016). Exercise for women receiving adjuvant therapy for breast cancer. The Cochrane Database of Systematic Reviews, 9, CDoo5001. https://doi.org/10.1002/14651858.CDoo5001.pub3

González-Robledo, L. M., González-Robledo, M. C., Nigenda, G., \& López-Carrillo, L. (2010). Acciones gubernamentales para la detección temprana del cáncer de mama en América Latina: Retos a futuro. Salud Pública de México, 52(6), 533-543.

Hacker, E. (2009). Exercise and Quality of Life: Strengthening the Connections. Clinical Journal of Oncology Nursing, 13(1), 31-39. https://doi.org/10.1188/o9.CJON.31-39

Holmes, M. D., Chen, W. Y., Feskanich, D., Kroenke, C. H., \& Colditz, G. A. (2005). Physical Activity and Survival After Breast Cancer Diagnosis. JAMA, 293(20), 2479-2486. https://doi.org/10.1001/jama.293.20.2479

Hong, S., Bardwell, W. A., Natarajan, L., Flatt, S. W., Rock, C. L., Newman, V. A., ... Group, F. the W. H. E. and L. (WHEL) S. (2007). Correlates of physical activity level in breast cancer survivors participating in the Women's Healthy Eating and Living (WHEL) Study. Breast Cancer Research and Treatment, 101(2), 225-232. https://doi.org/10.1007/s10549-006-9284-y

Irwin, M. L., McTiernan, A., Bernstein, L., Gilliland, F. D., Baumgartner, R., Baumgartner, K., \& Ballard-Barbash, R. (2004). Physical activity levels among breast cancer survivors. Medicine and science in sports and exercise, 36(9), 1484-1491.

Kang, K. D., Bae, S., Kim, H.-J., Hwang, I. G., Kim, S. M., \& Han, D. H. (2017). The Relationship between Physical Activity Intensity and Mental Health Status in Patients with Breast Cancer. Journal of Korean Medical Science, 32(8), 1345-1350. https://doi.org/10.3346/jkms.2017.32.8.1345

Mohammadi, S., Sulaiman, S., Koon, P. B., Amani, R., \& Hosseini, S. M. (2013). Impact of healthy eating practices and physical activity on quality of life among breast cancer survivors. Asian Pacific Journal of Cancer Prevention: APJCP, 14(1), 481-487. Recuperado de http://journal.waocp.org/?sid=Entrez:PubMed\&id=pmid:23534778\&key=2013.14.1.481

Mutrie, N., Campbell, A. M., Whyte, F., McConnachie, A., Emslie, C., Lee, L., ... Ritchie, D. (2007). Benefits of supervised group exercise programme for women being treated for early stage breast cancer: pragmatic randomised controlled trial. BMJ, 334(7592), 517. https://doi.org/10.1136/bmj.39094.648553.AE

OMS | La incidencia mundial del cáncer podría aumentar en un 50\% y llegar a 15 millones de nuevos casos en el año 2020. 
(s. f.). Recuperado 7 de mayo de 2018, de http://www.who.int/mediacentre/news/releases/2003/pr27/es/

OMS | Recomendaciones mundiales sobre la actividad física para la salud. (s. f.). Recuperado 7 de mayo de 2018 , de http://www.who.int/dietphysicalactivity/factsheet_recommendations/es/

Ortiz, A., Tirado, M., Hughes, D. C., Gonzalez, V., Song, J., Mama, S. K., \& Basen-Engquist, K. (2018). Relationship between physical activity, disability, and physical fitness profile in sedentary Latina breast cancer survivors. Physiotherapy Theory and Practice, 1-12. https://doi.org/10.1080/09593985.2018.1424978

Ramírez, K., Acevedo, F., Herrera, M. E., Ibáñez, C., \& Sánchez, C. (2017). Physical activity and breast cancer. Revista médica de Chile, 145(1), 75-84. https://doi.org/10.4067/So034-98872017000100011

Registro Poblacional de Cáncer de Cali. (s. f.). Recuperado 1 de noviembre de 2018, de http://rpcc.univalle.edu.co/es/ Mortalidad/Estadisticas/index.php

Sabino Neto, M., Moreira, J. R., Resende, V., \& Ferreira, L. M. (2012). Nível de atividade física em mulheres mastectomizadas e submetidas a reconstrução mamaria. Revista Brasileira de Cirurgia Plástica, 27(4), 556-561. https://doi. org/10.1590/S1983-51752012000400015

Salas Zapata, C., \& Grisales Romero, H. (2010). Calidad de vida y factores asociados en mujeres con cáncer de mama en Antioquia, Colombia. Revista Panamericana de Salud Pública, 28, 9-18. https://doi.org/10.159o/S1020-49892010000700002

Vardar-Yagli, N., Sener, G., Saglam, M., Calik-Kutukcu, E., Arikan, H., Inal-Ince, D., ... Kaya, E. B. (2015). Associations among physical activity, comorbidity, functional capacity, peripheral muscle strength and depression in breast cancer survivors. Asian Pacific Journal of Cancer Prevention: APJCP, 16(2), 585-589.

\section{Notas}

El presente artículo hace parte del macroproyecto: "Relación entre fatiga y capacidad aeróbica en mujeres con cáncer de mama no metastásico en una institución de salud de alta complejidad del valle del cauca en el 2013-2014”, Comité de Ética de la Universidad del Valle (Acta de aprobación o21-012).

Información de autores:

\section{Paola Andrea Córdoba Barona}

Fisioterapeuta. Esp. en Fisioterapia Cardiopulmonar Fundación Clínica Valle de Lili, Cali, Colombia andrea.cordoba@correounivalle.edu.co https://orcid.org/oooo-ooo2-2636-6612

\section{Gina Paola Velasco Villani}

Fisioterapeuta

Fundación Clínica Valle de Lili, Cali, Colombia

https://orcid.org/oooo-0oo2-5695-5354

\section{Viviana Carolina López López}

Fisioterapeuta

Christus Sinergia Clínica Farallones, Cali, Colombia

https://orcid.org/oooo-0oo2-4560-1120

\section{Angelly Bustamente}

Fisioterapeuta. MSc. en Epidemiología

Universidad del Valle, Cali, Colombia

Escuela Nacional Del Deporte, Cali, Colombia

https://orcid.org/oooo-0003-3898-0879

\section{Esther Cecilia Wilches-Luna}

Fisioterapeuta. Esp. en Fisioterapia Cardiopulmonar Universidad del Valle, Cali, Colombia

Sociedad de Fisioterapeutas Respiratorios Sofire SAS https://orcid.org/oooo-0003-3255-7607 